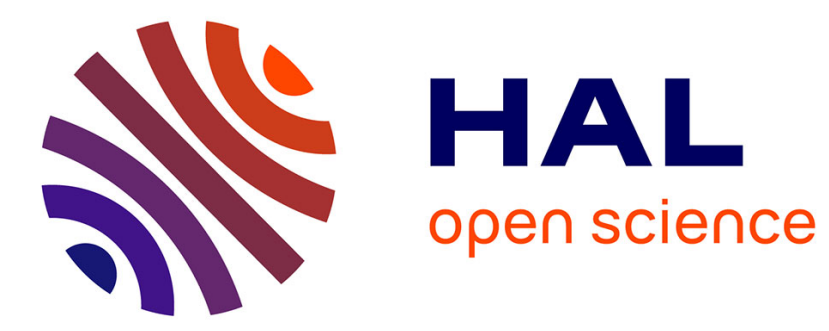

\title{
DNA I-motif provides steel-like tough ends to chromosomes
}

\author{
Raghvendra Pratap Singh, Ralf Blossey, Fabrizio Cleri
}

\section{To cite this version:}

Raghvendra Pratap Singh, Ralf Blossey, Fabrizio Cleri. DNA I-motif provides steel-like tough ends to chromosomes. Materials Research Society Fall Meeting (MRS Fall 2013), Dec 2013, Boston, United States. pp.135-141, 10.1557/opl.2014.282 . hal-00944020

\section{HAL Id: hal-00944020 \\ https://hal.science/hal-00944020}

Submitted on 27 Jul 2021

HAL is a multi-disciplinary open access archive for the deposit and dissemination of scientific research documents, whether they are published or not. The documents may come from teaching and research institutions in France or abroad, or from public or private research centers.
L'archive ouverte pluridisciplinaire HAL, est destinée au dépôt et à la diffusion de documents scientifiques de niveau recherche, publiés ou non, émanant des établissements d'enseignement et de recherche français ou étrangers, des laboratoires publics ou privés. 


\title{
DNA i-motif provides steel-like tough ends to chromosomes
}

\author{
Raghvendra P. Singh ${ }^{1,2}$, Ralf Blossey ${ }^{2}$ and Fabrizio Cleri ${ }^{1}$ \\ ${ }^{1}$ Institut d'Electronique Microelectronique et Nanotechnologie (IEMN Cnrs - UMR 8520), \\ University of Lille I Sciences and Technology, 59652 Villeneuve d'Ascq, France \\ ${ }^{2}$ Interdisciplinary Research Institute (IRI Cnrs - USR 3078), University of Lille I Sciences and \\ Technology, 59655 Villeneuve d'Ascq, France
}

\begin{abstract}
We studied the structure and mechanical properties of DNA i-motif nanowires by means of molecular dynamics computer simulations. We built up to $230 \mathrm{~nm}$-long nanowires, based on a repeated $\mathrm{TC}_{5}$ sequence from NMR crystallographic data, fully relaxed and equilibrated in water. The unusual $\mathrm{C} \cdot \mathrm{C}+$ stacked structure, formed by four ssDNA strands arranged in an intercalated tetramer, is here fully characterized both statically and dynamically. By applying stretching, compression and bending deformations with the steered molecular dynamics and umbrella sampling methods, we extract the apparent Young's and bending moduli of the nanowire, as well as estimates for the tensile strength and persistence length. According to our results, i-motif nanowires share similarities with structural proteins, as far as their tensile stiffness, but are closer to nucleic acids and flexible proteins, as far as their bending rigidity is concerned. Curiously enough, their tensile strength makes such DNA fragments tough as mild steel or a nickel alloy. Besides their yet to be clarified biological significance, i-motif nanowires may qualify as interesting candidates for nanotechnology templates, due to such outstanding mechanical properties.
\end{abstract}

\section{INTRODUCTION}

The DNA i-motif is one of the recently identified, non-standard DNA structures, which do not follow the standard Watson-Crick association rule [1-6]. In-vitro, under acidic $\mathrm{pH}$ conditions, the i-motif exists as a tetrameric structure, formed by four intercalated DNA strands, held together by protonated cytosine-cytosine, or $\mathrm{C} \cdot \mathrm{C}+$, pairs. However, $\mathrm{i}-$ motif tetramers have also been observed in vivo, most notably in the terminal part of the human genes, or telomere, where rather long (50-210 bases) asymmetric G-rich and C-rich single-stranded portions of DNA are found $[5,6]$. Besides their possible role in the genome, still awaiting a full clarification, such DNA nanowires can be also attractive in the domain of bio-inspired materials for nanotechnologies [7-10]. Notably, various kinds of biomimetic nanowires have been already obtained from B-DNA, proteins, and even from viral particles. Electrical, optical, plasmonic features have been added to such wires by metallization, wherein metals have been "coated" or "moulded" onto the outer or inner surfaces of these biomolecular templates $[11,12]$. The i-motif could as well be a good candidate for nano-templating, being easily be manipulated and apparently stable over quite long time scales. However, while its molecular structure is rather well assessed, a thorough mechanical characterization of such bionanowire is still lacking. In a recent work [13] we calculated the main elastic moduli (Young's modulus, bending stiffness, persistence length) by means of Molecular dynamics computer 
simulations. In the present note, we summarize such results, and extend our investigation to the determination of the mechanical toughness of the DNA i-motif. We highlight the very peculiar character of this supramolecular structure, and speculate about the potential biological and genetic implications of this material, displaying a combination of high toughness, high strength, and good flexibility.

\section{THEORY}

Mechanical characterization of individual molecules can be performed and explored by single-molecule pulling experiments, in which molecules are stretched under the influence of a mechanical force, for example by an atomic force microscope (AFM) [14]. The detailed molecular level understanding of such pulling experiments can be greatly enhanced by the use of atomistic simulations. Steered Molecular Dynamics (SMD, [15]) is one of the best available tools, commonly used in such studies, to deduce the mechanical response of molecules subject to an external force.

In a classical AFM experiment, one end of the molecule is attached to a cantilever and the other end of the molecule is attached to a surface. Then, the cantilever is moved away from the surface with the constant velocity $v$, and the force response of the molecule can be measured, as being equal and opposite to the force response of the cantilever.

Similarly to what happens in a AFM experiment, in SMD simulations one end of the molecule is attached to a virtual spring, with a force constant $k_{c}$, and the other end is fixed by a harmonic restraint potential. By analogy with the AFM, the mechanical response of the molecule can be measured by moving the spring. The force response of the molecule can be calculated by the relation between spring constant and extension:

$$
f=k_{c}\left(R_{0}-R\right)
$$

where $R_{0}$ is the total length, that is the contour length (distance between the two ends of the molecule) plus the extension of the spring, and $R$ is the actually simulated extension of the molecule itself. The total extension is usually represented by a linear rate as:

$$
R_{0}=R_{\text {fold }}+v t
$$

where $R_{\text {fold }}$ is the initial extension in the folded state, $v$ is the stretching velocity, and $t$ is the time. The output of the single SMD simulation is a force-extension curve $f(R)$ of the molecule for a given stretching velocity.

In this work, SMD simulations were performed to study the elasticity under stretching and bending of the hydrated $\mathrm{i}$-motif structures, using the constant-velocity or constant-force protocols of NAMD [16]. To perform SMD, four atoms (the terminal C of each strand of the i-motif tetramer) were fixed at one end of the structure. Similarly, at the opposite end, four atoms were attached to the harmonic potential, carrying the applied constant velocity. Under the applied perturbation at one end, while holding still the other end, the i-motif structure responds by developing a steady deformation, tension, compression, or bending, according to the eigenvector of the applied perturbation. The center of pull was calculated by averaging the coordinates of all the SMD atoms. The direction of pull was identified by the direction of the vector connecting the fixed and moving atoms. The center of pull of the moving atoms was attached to a dummy atom by a virtual harmonic spring. The 
constant velocity along the $\mathrm{z}$-axis was applied to the dummy atom and the force drop between the two ends was measured. After several tests, a virtual spring constant of $1 \mathrm{kcal} \mathrm{mol}^{-1} \AA^{-2}$ was adopted together with typical pulling speeds of 1 to $3 \mathrm{~ms}^{-1}$, to ensure a good signal-to-noise ratio.

Bending was simulated by means of the "umbrella sampling" technique [17]. A harmonic biasing potential was used, only to simulate the action of a force at the midpoint of the nanowire (the central nucleotide tetrad, one base per chain), while holding still the two extremes. In this way we could drive the nanostructure along the bending deformation trajectory, with a pulling velocity of 5 $\mathrm{ms}^{-1}$ up to reaching the maximum deformation of $5 \mathrm{~nm}$. The umbrella potential provided the confinement in the bent structure, while exploring the most stable conformations to extract the corresponding values of deformation energy and force along the trajectory.

\section{DISCUSSION}

\section{Structural stability of the DNA i-motif}

After a first round of classical MD simulations, we obtained the equilibrium structure of the DNA i-motif, $n\left[\left(\mathrm{TC}_{5}\right)_{4}\right]$, constructed by the periodic repeat of the base $\left[\left(\mathrm{TC}_{5}\right)_{4}\right]$ tetramer unit from the NMR structure [3]. Several indicators of the structural stability were assessed (Figure 1): average interplanar and intercalation base-pair distance (i.e., within each strand, and between intercalated strands, respectively); average helical twist $\left(\sim 12^{\circ}\right.$, compared to $\sim 34^{\circ}$ in B-DNA); radial distribution

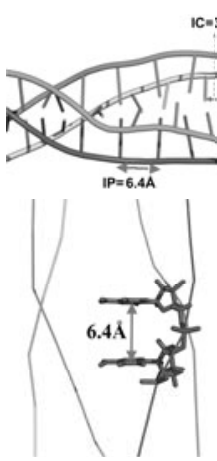

B
$D=18.5 \mathrm{~A} \quad M i G=7.9 \mathrm{~A}$

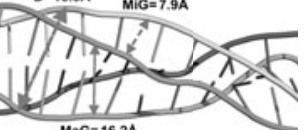

A

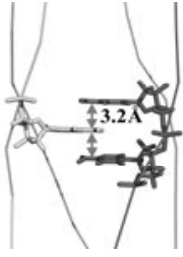

C

Figure 1: Definition of the various distances, of the nucleotides, in the four chains composing the i-motif tetramer. Average values obtained after the relaxationequilibration molecular dynamics cycle described in the text. (A) The four strands show the intercalated structure of the i-motif, a tetramer composed by four independent and symmetric ssDNA strands; $\mathrm{IP}=$ interplanar (or base stacking) distance (see also (B)); IC=intercalation distance (see also (C)); $\mathrm{D}=$ diameter; $\mathrm{MiG}=$ minor groove width; $\mathrm{MaG}=$ major groove width.

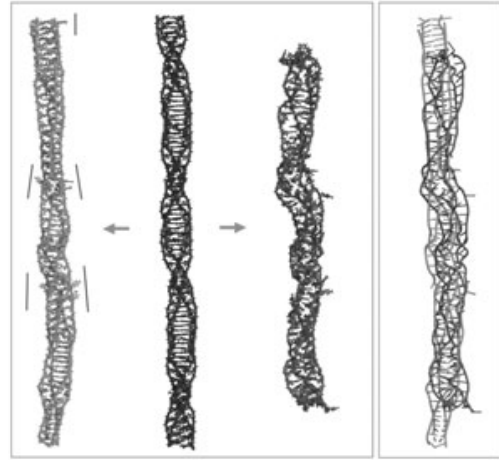

A

B

C

D

Figure 2: Comparison of the structure of the protonated vs. non-protonated i-motif tetramer after $5 \mathrm{~ns}$ of molecular dynamics at $\mathrm{T}=300 \mathrm{~K}$. The central (B) structure in blue is the initial configuration, identical for both cases; (B) the protonated tetramer (thin bars indicate the outwards leaning Thymine bases), evolving into the correct $\mathrm{i}$-motif structure; (C) the non-protonated tetramer, evolving into a disordered structure; (D), the two structures superimposed. 
of water molecules and ions $\left(\mathrm{Na}^{+}\right.$and $\mathrm{Cl}^{-}$); sugar pucker of the ribose ring (strictly $\mathrm{C} 3$ '-endo, compared to a mix of C2'- and C3'-endo in B-DNA); conformation of the T's, alternately leaning inwards and outwards the main cylindrical axis in agreement with experimental observation.

However, the chief indicator to demonstrate the dynamical stability of the structure was the study of the role of the resonant protonation. The comparison between the dynamical evolution of the fully protonated $(n=8) 8\left[\left(\mathrm{TC}_{5}\right)_{4}\right] \mathrm{i}-$-motif and the non-protonated $(\mathrm{np})$ tetramer with the same nucleotide composition, $8\left[\left(\mathrm{dTC}_{5}\right)_{2}\right]$, was performed, over a time scale of $5 \mathrm{~ns}$ (in practical terms, the two starting structure were identical, apart from the extra proton placed between $\mathrm{N} 3$ nitrogen atoms of the $\mathrm{C} \cdot \mathrm{C}$ pairs.). Figure 2 shows a comparison of the structures of the $\mathrm{i}-\operatorname{motif}(2 . \mathrm{A})$ and the $\mathrm{np}-$ tetramer (2.C), starting from the same initial, unrelaxed 8-unit configuration (2.B). It is found that during this time, the np-tetramer crumbles, and loses the $\mathrm{i}$-motif-like initial structure, becoming a rather disordered structure with a wide distribution of different interplanar distances for every base pair. By contrast, the protonated i-motif maintains a linear and straight backbone, with constant interplanar and intercalation distances, eventually adjusting to the correct, straight conformation of the well- equilibrated i-motif, and reorganizing the initial defects introduced by the artificial periodic construction. Such findings clearly support the idea of the key role of Cytosine protonation, in establishing the dynamically stable form, and structural integrity of the $\mathrm{i}$-motif structure.

\section{Mechanical properties of the DNA i-motif}

We started our simulations from a fully extended $8\left[\left(\mathrm{TC}_{5}\right)_{4}\right]$ i-motif nanowire, with contour length $\mathrm{L}=28.8 \mathrm{~nm}$, to which the tensile deformation was applied by SMD. Figure 3 shows the typical result of a uniaxial deformation experiment for the $8\left[\left(\mathrm{TC}_{5}\right)_{4}\right]$ i-motif, both in compression and in tension. The force-displacement plot under such conditions is indeed quite noisy, and displays a moderately oscillatory shape due to the non-homogeneous (also in time) molecular relaxation. However, it should be noted that the relative deformation $\Delta L / L$ over which the elastic modulus is computed is extremely small, for a typical MD simulation -0.1 to $+0.3 \%$ at the slower deformation rate. In the approximation of the nanowire as a linear-elastic rigid rod of uniform density, the Young's modulus $Y$ can be extracted from the linear fit of the $f$ vs. $\Delta L$ data in Figure 3, as:

$$
\frac{f}{A}=Y\left(\frac{\Delta L}{L}\right)
$$

where $A=\pi R^{2}$ is the cross section of the nanowire (assumed cylindrical), $R=0.95 \mathrm{~nm}$. A clear signature of the truly linear-elastic response is the symmetry between tension and compression (positive and negative $\Delta L$ ), meaning that no internal structural modifications should be induced by the deformation. The best estimate extracted from the linear fit is $Y=1.8 \pm 0.5 \mathrm{GPa}$, a quite large value compared to the Young's modulus of B-DNA, which lies rather in the range of $0.35 \mathrm{GPa}$ [18].

The apparent toughness of the $i$-motif could be probed by stretching the nanowire well beyond the linear elastic regime. Of course, with the Amber force field employed in our SMD simulations it is, by construction, impossible to break chemical bonds. However, an upper limit to the mechanical yielding can at least be estimated, by stretching up to the first evidences of internal structural collapse of the molecular structure. We performed extreme elongations $\Delta L$ of the $8\left[\left(\mathrm{TC}_{5}\right)_{4}\right]$ $i$-motif, without detecting any signs of mechanical instability up to forces of the order of $1000 \mathrm{pN}$ $(\Delta L / L \sim 20 \%)$ and more. Assuming a simplified definition of the principal tensile stress, averaged 
over all the perpendicular components, and in the absence of shearing, as $\left\langle\sigma_{3}\right\rangle=f / A$, the tensile strength $\sigma_{t}$ (the peak value of tensile stress in the linear region of the stress-strain curve, prior to yielding) of the $i$-motif would exceed $100 \mathrm{MPa}$. Such a value of $\sigma_{t}$ allows to estimate a mechanical toughness, $\tau$, defined as the area under the stress-strain curve from 0 to $\sigma_{t}$ :

$$
\tau=\int_{0}^{\sigma t} \sigma_{3}(\varepsilon) d \varepsilon
$$

with the uniaxial strain $\varepsilon=\Delta L / L$. According to the above values, we estimate a $\tau \sim 300 \mathrm{MPa}$, or more, notably as good as a mild steel or an aluminum alloy wire. Also in this respect, the $i$-motif appears to differ substantially from B-DNA, which is known to undergo a kind of structural, or "melting" transition above a tensile force of just $\sim 65 \mathrm{pN}$ [18], a fact that also complicates the direct experimental measurement of $Y$.

For a purely homogeneous cylindrical beam (or wire), the critical load to the Euler buckling instability is given as [19]:

$$
F_{\text {crit }}=\frac{4 \pi^{2} B}{L^{2}}
$$

The mechanical meaning of the bending stiffness $B$ in Eq. (5) can be appreciated by calculating the energy required to bend a wire of length $L$ by an angle $\theta$. If for simplicity we assume a constant curvature $\gamma=d \theta / d l$, with the radius of curvature defined as $\rho=1 / \gamma$, the bending energy (at

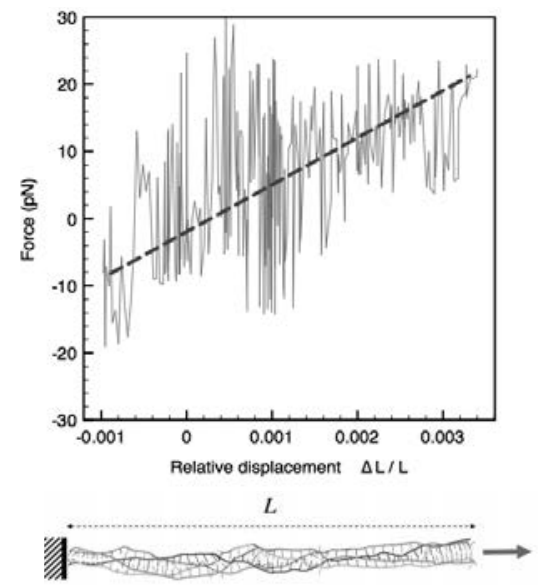

Figure 3: Force-displacement plot for uniaxial stretching deformation of the $i$-motif tetramer. Results for a constant pulling velocity of $1 \mathrm{~ms}^{-1}$ and a spacer spring constant of $1 \mathrm{kcal} \mathrm{mol}^{-1} \mathrm{~A}^{-2}$. The blue dashed line is the linear fit to the average mechanical response. Below: a schematic of the stretching simulation.

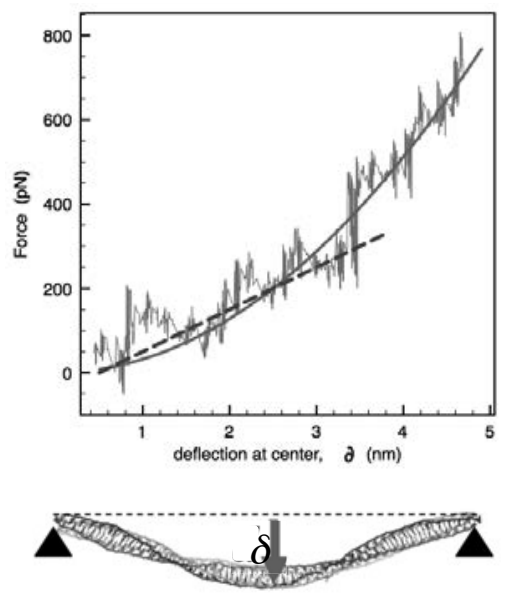

Figure 4: Force-displacement plot for the bending deformation of the $i$-motif tetramer. The red continuous line is a guide to the eye (quadratic fit); the thick-dash blue line is the best linear fit at small bending. Below: a schematic of the bending simulation. 
constant $\rho=L / \theta)$ is [19]:

$$
E_{\text {bend }}=\frac{B}{2} \int_{0}^{L}\left(\frac{d \theta}{d l}\right)^{2} d l=\frac{B L}{2 \rho^{2}}=\frac{B \theta^{2}}{2 L}
$$

$l \in[0, L]$ being a variable spanning the contour length $L$ of the wire. In free fluctuation, the thermal force $F_{t h} \sim k_{B} T / L$ acts as a random load, with (random) compressive and bending components, whose energy is to be compared to the bending energy. For example, to bend by $\theta=30^{\circ}$ a nanowire of $L=30$ $\mathrm{nm}$, with a typical $B$ value for stiffer biological polymers of $\sim 10^{-26} \mathrm{~J} \mathrm{~m}$, the energy required according to Eq. (6) is $E_{\text {bend }} \sim 10^{-19} \mathrm{~J}$. Since $k_{B} T=4.14 \cdot 10^{-21} \mathrm{~J}$ at room temperature, it appears that only quite large thermal fluctuations, i.e. very long observation times, could lead to a reliable estimate of $B$ by studying the fluctuation of $L$.

A more reliable estimate of $B$ can be obtained from the calculation of the displacement $\delta$ at the midpoint produced by a point load $f$, as the derivative of the elastic energy with respect to the load (beam theory, Castigliano's theorem [19]):

$$
\delta=\frac{d}{d f}\left(\int_{-L / 2}^{L / 2} \frac{M^{2}(l)}{2 B} d l\right)=\frac{f L^{3}}{48 B}
$$

$l \in[0, L]$ being a variable spanning the contour length $L$ of the wire, and $M(l)=\frac{1}{2} f \cdot l$ the bending moment, assumed linear along $L$ for a straight wire. Therefore, from the fit of the linear part of the force plot as a function of $\delta$ in Figure 4, we get: $B=2.6 \pm 0.4 \cdot 10^{-26} \mathrm{~N} \mathrm{~m}^{2}$.

Moreover, we can also estimate the persistence length from the relationship [13]:

$$
\lambda_{P}=\frac{B}{k_{B} T}=4 \pm 2 \mu \mathrm{m}
$$

The DNA i-motif appears to belong with the class of structural polymers (such as F-actin, microtubules, keratin, etc.), as far as its Young's modulus is concerned. Moreover, it appears a nearly metallic material with its tensile strength $\sigma_{t}>100 \mathrm{MPa}$ and toughness $\tau>300 \mathrm{MPa}$. Notably, with a value of $Y$ in the GPa range, the $\mathrm{i}-$ motif is at least one order of magnitude stronger than the DNA strands by which it is made up. On the other hand, if we look at its transverse flexibility as measured by the bending stiffness $B$ or the persistence length $\lambda_{p}$, the i-motif seems closer to DNA, and 2-3 orders of magnitude more flexible than F-actin or microtubules.

\section{CONCLUSIONS}

The presence of i-motif structures at the promoter regions of many oncogenes points out their potential biological significance [20-22]. Since C-rich DNA regions form i-motif structures, the opposite strand of DNA should have, in theory, the tendency to form a G-quadruplex. Such higherorder DNA structures cannot bind with proteins, nor can form active complexes for transcription, resulting in down-regulation of the close-by genes. This feature would make them a drug target of high importance.

As a relatively flexible, but very stiff and very tough nanostructure at the same time, i-motif fragments in the centromere or telomere regions of the genes could impart an unusually high 
toughness and rigidity to some portions of the genome, suggesting some speculations about the genetic meaning, or function, of such features. Just for the sake of argument, telomere length in white blood cells has been repeatedly observed to have an inverse correlation with blood pressure [23]; it is directly related with loss of elasticity of arterial wall [24]; and, its shortening is increasingly accepted as a predictive biomarker for cardiovascular disease [25]. It is highly speculative to ask whether such effects in leucocytes could also have a mechanical component, stemming from a higher rigidity of telomere regions, ultimately linked to the possible presence of i-motif structures. If, at least partly, chromatin integrity could be associated with the presence of long telomeres, one may wonder whether the age-related, progressive shortening of telomeres could also imply the absence of tougher i-motifs segments, someway contributing to cell aging via easier DNA degradation.

\section{ACKNOWLEDGMENTS}

Computing grants from the French Supercomputing Center IDRIS and CEA-TGCC in the frame of the PRACE 2010-030294 Project to FC and RB, are acknowledged. RPS gratefully thanks the President of the University Lille I for a collaborative, three-year PhD grant.

\section{REFERENCES}

1. J. L. Leroy, M. Gueron, J. L. Mergny and C. Helene, Nucl. Acids Res. 22 (1994) 1600

2. S. Nonin and J. L. Leroy, J. Mol. Biol. 261 (1996) 399

3. A. T. Phan and J. L. Leroy, J. Biomol. Struct. Dyn. 17 (2000) 245

4. K. S. Jin, S. R. Shin, B. Ahn, Y. Rho, S. J. Kim and M. Ree, J. Phys. Chem. B 113 (2009) 1852

5. J. Choi, S. Kim, T. Tachikawa, M. Fujitsuka and T. Majima, J. Am. Chem. Soc. 133 (2011) 16146

6. J. Smiatek, C. Chen, D. Liu and A. Heuer, J. Phys. Chem. B 115 (2011) 13788

7. Y. Wang, X. Li, X. Liu and T. Li, Chem. Commun. (Cambr) 42 (2007) 4369

8. Y. Peng, X. Wang, Y. Xiao, L. Feng, C. Zhao and J. Ren, J. Am. Chem. Soc. 131 (2009) 13813

9. X. Ren, F. He and Q. H. Xu, Chem. Asian. J. 5 (2010) 1094

10. C. Wang, Y. Du, Q. Wu, S. Xuan, J. Zhou and J. Song, Chem. Commun. (Cambr) 49 (2013) 5739

11. H. W. Fink and C. Schonenberger, Nature 398 (1999) 407

12. H. Yan, S. H. Park, G. Finkelstein, J. H. Reif and T. H. LaBean, Science 301 (2003) 1882

13. R. P. Singh, R. Blossey and F. Cleri, Biophys. J. 105 (2013)

14. M. Rief, M. Gautel, F. Oesterhelt, J. M. Fernandez and H. E. Gaub, Science 276 (1997) 1109

15. B. Isralewitz, M. Gao and K. Schulten, Curr. Opin. Struct. Biol. 11 (2001) 224

16. J. C. Phillips et al., J. Comput. Chem. 26 (2005) 1781

17. J. Kastner, J. Chem. Phys. 131 (2009) 034109

18. S. B. Smith, Y. Cui and C. Bustamante, Science 271 (1996) 795

19. J. M. Gere and S. P. Timoshenko, Mechanics of materials, Nelson Th., Cheltenham, UK, 1999.

20. K. Guo et al., J. Am. Chem. Soc. 129 (2007) 10220

21. N. Khan, A. Avin, R. Tauler, C. Gonzalez, R. Eritja and R. Gargallo, Biochimie 89 (2007) 1562

22. D. Sun and L. H. Hurley, J. Med. Chem. 52 (2009) 2863

23. E. Jeanclos et al., Hypertension 36 (2000) 195

24. Y. Y. Wang, H. Z. Wang, L. Y. Xie, K. X. Sui and Q.-Y. Zhang, The Aging Male 14 (2010) 27

25. J. Hoffmann and I. Spyridopoulos, Future Cardiology 7 (2011) 789 